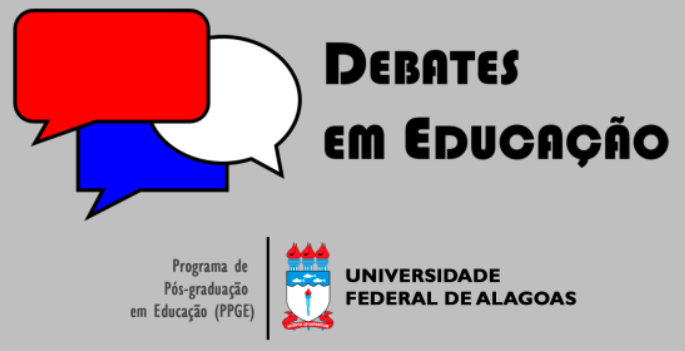

ISSN Eletrônico 2175-6600

Vol. I | | No. 23 | Jan./Abr. | 2019

\section{Maximiliano Rúa}

Universidad de Buenos Aires (UBA - Argentina) maximiliano.rua.74@gmail.com

\section{EL CURRÍCULUM DESDE UN ENFOQUE ANTROPOLÓGICO}

\section{RESUMO}

A partir de un enfoque que se propone; antropologizar los objetos tradicionalmente abordados por la didáctica (Cerletti y Rúa, 2017); abordamos el análisis del currículum, como producto sociocultural e histórico de una determinada sociedad. Este enfoque nos permite sostener que el currículum, las teorías curriculares y los diseños curriculares son una de las prácticas a partir de las cuales una determinada sociedad organiza la transmisión, apropiación y producción de los conocimientos que se consideran legítimos para las subsiguientes generaciones. Lo que implica analizar dichos objetos sociales -los currículums, los diseños curriculares y las teorías curriculares- cómo; parte de una determinada política estatal; una particular configuración disciplinar; y una determinada posición de clase social. En particular en esta presentación me propongo abordar hacer un breve repaso histórico de las diferentes conceptualizaciones sobre el currículum desarrolladas en los campos disciplinares afines. Posteriormente analizaremos las referencias que, tanto desde los diseños curriculares como desde las teorías curriculares, se construyen del concepto de "cultura" como categoría explicativa para la validación de determinados conocimientos sociales por sobre otros, lo que supone asimismo una manera de construir conocimiento sobre la propia sociedad. Analizar los usos históricos y actuales del currículum permite visibilizar como un determinado grupo organiza la transmisión, apropiación y producción de los conocimientos que se consideran legítimos en un determinado momento histórico para la propia sociedad. Finalmente, haremos una propuesta para abordar el currículum desde y para la formación de las y los antropólogas/os. Abriendo un debate en torno a la interdisciplinaridad y los desafíos que supone la enseñanza de la Antropología en contextos donde las prácticas de construcción de conocimiento son legitimadas a partir del uso que otros campos disciplinares (Pedagogía, Didáctica, etc.).

Palavras-chave: Currículum. Conocimiento. Categoría nativa y cultura.

\section{THE CURRICULUM FROM AN ANTHROPOLOGICAL PERSPECTIVE}

\begin{abstract}
From a proposed approach; anthropologizing the objects traditionally addressed by didactics (Cerletti and Rúa, 2017); we approach the analysis of the curriculum, as a sociocultural and historical product of a certain society. This approach allows us to maintain that the curriculum, curricular theories and curricular designs are one of the practices from which a certain society organizes the transmission, appropriation and production of the knowledge that is considered legitimate for subsequent generations. What it implies to analyze these social objects -the curricula, the curricular designs and the curricular theories- how; part of a specific state policy; a particular disciplinary configuration; and a certain position of social class. In particular in this presentation I intend to address a brief historical review of the different conceptualizations about the curriculum developed in the related disciplinary fields. Later we will analyze the references that, both from curricular designs and from curricular theories, are constructed from the concept of "culture" as explanatory category for the validation of certain social knowledge over others, which also supposes a way to build knowledge about the own society. Analyzing the historical and current uses of the curriculum allows to visualize how a certain group organizes the transmission, appropriation and production of the knowledge that is considered legitimate at a certain historical moment for society itself. Finally, we will make a proposal to address the curriculum from and for the training of anthropologists. Opening a debate around the interdisciplinarity and the challenges that the teaching of Anthropology supposes in contexts where the practices of construction of knowledge are legitimized from the use that other disciplinary fields (Pedagogy, Didactics, etc.).
\end{abstract}

Keywords: Curriculum. Knowledge. Native category and culture.

Submetido em: 16/12/2018

Aceito em: 03/04/2019

Ahead of print em: I I/04/2019

Publicado em: 25/04/2019 


\section{INTRODUCCIÓN}

"Como la navegación, la jardinería, la política y la poesía, el derecho y la etnografía son oficios de lugar: actúan a la luz del conocimiento local" (GEERTZ, I983 p. 194).

El estudio de las diferentes instituciones sociales, que una determinada sociedad despliega en un momento histórico y social, tiene una rica y extensa tradición en la Antropología ${ }^{1}$. Desde los trabajos pioneros de Bronislaw Malinowski en las Islas Trobriand analizando el kulay los estudios del don de Marcel Mauss pasando posteriormente por el registro de Evans-Pritchard sobre el tiempo entre los Nuer o las reflexiones de Radcliffe-Brown respecto al lugar que los rituales tienen en la conservación del "orden social"; continuando con las redefiniciones disciplinares que en la década del 80 Clifford Geertz lleva adelante explorando "el significado" de la "riña de gallos" o los planteamientos de Eric Wolf sobre como los pueblos no europeos estaban inmersos en procesos sociales que excedían lo local, hasta las "estructuras subyacentes" que para Lévi-Strauss configuran toda institución social²; la Antropología se ha propuesto analizar las diferentes prácticas a partir de las cuales una sociedad en un determinado momento histórico construye conocimiento respecto a si misma.

Una de las instituciones centrales en nuestra sociedad es el sistema educativo nacional obligatorio; y uno de los objetivos primordiales de dicho sistema es la transmisión, apropiación y producción ${ }^{3}$ de los conocimientos considerados necesarios y legítimos por dicha sociedad. Determinados conocimientos -de "hombre", de "comunidad", de "historia", etc.- son transmitidos por los sistemas escolares nacionales como universales, como expresión totalizante de una determinada sociedad. Unas de las prácticas más extendidas destinadas a construir conocimiento sobre la propia sociedad, por parte del sistema educativo, son aquellas que la Didáctica y/o la Pedagogía agrupan en lo que se conceptualiza bajo la categoría de currículum. A partir de un enfoque que se propone antropologizar los objetos tradicionalmente abordados en el campo de la didáctica y la pedagogía (CERLETTI Y RÚA, 20 I7); en este articulo analizaremos el currículum como

\footnotetext{
I Es importante aclarar que simultáneamente que la Antropología registra y conceptualiza las diferentes prácticas a partir de las cuales una sociedad construye conocimiento sobre si misma está problematizando las propias prácticas, tanto disciplinares como sociohistóricas de construcción de conocimiento sobre su sociedad.

${ }^{2}$ Bronisław Kasper Malinowski ( 884 - 1942) Fue uno de los referentes centrales de la antropología social británica. El Kula es un intercambio ceremonial que se realiza entre los pobladores de las islas Trobriand. Marcel Mauss ( I872-1950) es uno de los referentes de la "etnología francesa". El don crea un vínculo social entre quien lo ofrece y lo recibe. Edward Evans-Pritchard (1902-1973) fue un antropólogo inglés central de la consolidación de la antropología social británica El tiempo en la visión de mundo nuer se basan en la relación que hay entre los distintos grupos sociales, que se están moviendo al mismo tiempo por un mismo contexto. Alfred Reginald Radcliffe-Brown (I 88 I - I 955) fue un antropólogo inglés a quien se debe el desarrollo del funcionalismo estructuralista. Clifford James Geertz (1926-2006) Fue un antropólogo estadounidense, clave en el desarrollo de la Antropología Interpretativa."En la riña de gallos, el balinés forma y descubre su temperamento y al mismo tiempo el temperamento de su sociedad". (Geertz, 1973, p 37 I). Eric Robert Wolf (1923-1999) Fue un antropólogo e historiador estadounidense de corte marxista. Claude Lévi-Strauss (1908-2009) Fue un antropólogo y etnólogo francés, fundador de la antropología estructural.

${ }^{3}$ La transmisión da cuenta del conjunto de prácticas a partir de las cuales adultos y niños comparten entre sí conocimientos constitutivos de la vida en sociedad en cada período histórico; o los contenidos desplegados en las situaciones escolares. (RÚA, 20 I7).Al analizar las relaciones a partir de las cuales los sujetos hacen suyos los conocimientos constitutivos de la vida en sociedad, transformando sus propios conocimientos, sostenemos que se está desplegando un proceso de apropiación. (RÚA, 20 17).A las prácticas desplegadas entre pares, o con los miembros de otras generaciones, que crean conocimientos las consideramos un proceso de producción. (RÚA, 2017).
} 
una de las diferentes prácticas a partir de las cuales una determinada sociedad construye conocimiento sobre sí misma en determinadas circunstancias históricas.

En primer término, haremos un breve repaso histórico de las diferentes conceptualizaciones sobre el currículum desarrolladas en los campos disciplinares afines. Posteriormente reflexionaremos sobre las referencias que, tanto desde los diseños curriculares como desde las teorías curriculares, se construyen del concepto de "cultura" como categoría explicativa para la validación de determinados conocimientos sociales por sobre otros, lo que supone asimismo una manera de construir conocimiento sobre la propia sociedad. Analizar los usos históricos y actuales del currículum permite visibilizar como un determinado grupo organiza la transmisión, apropiación y producción de los conocimientos que se consideran legítimos en un determinado momento histórico para la propia sociedad. Finalmente, haremos una propuesta para abordar el currículum desde y para la formación de las y los antropólogas/os. Abriendo un debate en torno a la interdisciplinaridad y los desafíos que supone la enseñanza de la Antropología en contextos donde las prácticas de construcción de conocimiento son legitimadas a partir del uso que otros campos disciplinares (Pedagogía, Didáctica, etc.).

\section{EL CURRÍCULUM EN CLAVE HISTÓRICA: LAS TEORÍAS Y LOS DISEÑOS CURRICULARES}

Actualmente las Cs. de la Educación y/o la Pedagogía distinguen dos tipos de prácticas relacionadas con el currículum; las asociadas a los diseños curriculares, que condensan un conjunto de procedimientos didácticos, políticos, administrativos, económicos, etc. y otras relacionadas a la teoría curricular. El primer conjunto de prácticas hace referencia al uso; desde la elaboración de un documento escrito que llevan adelante los diferentes gobiernos -nacional, provincial, etc.- y que contiene objetivos, contenidos y criterios pedagógicos y didácticos destinadas a guiar la práctica de los diferentes sujetos. El segundo hacer referencia a los metadiscursos que componen el propio campo de estudio curricular. A continuación, haremos un breve repaso de algunas de las conceptualizaciones que desde diferentes disciplinas se ha hecho del currículum.

Es en la primera mitad del siglo $X X$, a partir de la expansión de la escolaridad de masas, la industrialización de la economía a gran escala y las transformaciones políticas y sociales, que surgen las primeras conceptualizaciones sobre el currículum. Dos perspectivas pedagógicas se conforman a principios de SXX; una "pedagogía industrial" representada por Franklin Bobbit quien publica en 1918 "The curriculum"4, y la "Nueva Escuela" de John Dewey esbozada en "The child and the curriculum" (1902).

\footnotetext{
${ }^{4}$ Posteriormente este enfoque es complejizado por Chárter que en 1924 escribe "Curriculum Construction".
} 
El enfoque de Bobbit ubicaba en el centro de su propuesta a los contenidos y la necesidad de establecer secuencias y jerarquizaciones en el currículum de lo que debe ser enseñado, y por ende de lo que se consideraba más o menos valido respecto al conocimiento de la propia sociedad. Dewey, quien fuera un crítico de la sociedad industrial -a quien se atribuye la metodología de "proyectos" actualmente en el centro de la escena de las modas pedagógicas- propone una una pedagogía basada en la categoría de "experiencia"; el currículum es todo aquello que sucede en la escuela y debe organizarse a partir de la experiencia de los diferentes sujetos escolares.

Al finalizar la $2^{\circ}$ Guerra Mundial Ralph Tyler publica "Principios básicos del currículum" (1949), en un intento -no logrado- por unificar las dos perspectivas; la iniciada por Dewey, centrada en la experiencia del escolar, y la desarrollada por Bobbit y Charter, centrada en la definición formal y organización de los contenidos a enseñar. El enfoque de Tyler ha sido, y sigue siendo, hegemónica tanto en los diseños curriculares como en la teoría curricular. Para Tyler el currículum es una herramienta diseñada por especialistas, expresa la cultura de una determinada sociedad, y guía las decisiones que se toman en relación en los diferentes espacios formativos. Ángel Diaz de Rada sostiene que “...la visión que estableció Tyler prevaleció en el campo casi por dos décadas, se internacionalizó en la década de los setenta conformando una generación de modelos pedagógicos que influyeron en las reformas educativas de esos años en América Latina..." (DIAZ DE RADA, 2003, p.4). Esta “internacionalización” del currículum estableció en los sistemas educativos una determinada práctica de construcción de conocimiento de la propia sociedad por sobre otra; un enfoque tecnicista y utilitario que privilegió prácticas de selección, jerarquización, secuenciación por sobre las que se proponían la recuperación de las diversas experiencias de la propia sociedad; privilegiando la formación de los sujetos como "ciudadanos", "trabajadores" de una "democracia" para caracterizar a la sociedad.

No obstante, a partir de la década del 70 comienzan a surgir cuestionamientos desde la propia teoría curricular a la perspectiva técnica que resultaba hegemónica en los diseños curriculares, principalmente a partir de las investigaciones de Philip Jackson quien en 1968 pública "La vida en las aulas". Jackson sostiene que las y los estudiantes aprenden en la cotidianeidad escolar un conjunto de reglas, normas, sentimientos, valores, diríamos desde la antropología prácticas que no son "declaradas" en los diseños curriculares oficiales, a este conjunto de "aprendizajes" el autor los denomina "currículum oculto". (JACKSON, 1968). Posteriormente Lawrence Stenhouse (1975), Michael Apple (1979) y Henry Giroux ( 1983 ) -entre otros- comienzan a problematizar el aspecto ideológico y las relaciones de poder implicadas tanto en los diseños curriculares como en las diferentes teorías curriculares.

"A este respecto es básico problematizar las formas de currículo que se encuentran en las escuelas a fin de poder descubrir su contenido ideológico latente. Hay que tomar muy en serio las siguientes preguntas sobre la tradición selectiva. ¿De quién es el conocimiento? ¿Quién lo seleccionó? ¿Por qué se organizó y enseñó de este modo? ¿Y por qué a este grupo particular? No 
basta, sin embargo, con el simple hecho de formular esos interrogantes. Hay que tratar también de vincular estas investigaciones con las concepciones en competencia de las ideologías y el poder social y económico. De ese modo se puede empezar a obtener una valoración más concreta de las vinculaciones existentes entre el poder económico y político y el conocimiento puesto a disposición (y el no puesto a disposición) de los estudiantes." (APPLE, 1979, p I6).

Stenhouse sostiene que el currículum es un "puente" entre los "principios" -los aspectos teóricosy la práctica educativa, lo que le permite afirmar que la dicotomía entre teoría y práctica no está presente en las aulas al momento que se despliega el currículum. Es importante destacar cómo los diferentes autores, por más críticos que sean de los diseños curriculares, al desarrollar sus teorías curriculares proponen un diseño curricular. En este sentido, autores como Gimeno Sacristán (2010) plantean que el currículum es un concepto de uso generalizado en el campo educativo, presente en la investigación, en los documentos de las políticas educativas, en los medios de comunicación y en los profesorados. El autor plantea "la existencia" de diferentes niveles de "concreción curricular"; el prescripto5; el presentado a las y los profesores (Diseños Curriculares, Manuales, Cursos de Formación, etc.); el apropiado por las y los profesores; en acción; el realizado (relacionado con los métodos pedagógicos); el evaluado; el ausente ("aquello que la escuela no enseña" (Eisner, 1985)); y el oculto. El mismo autor sostiene que el currículum supone "el contenido cultural que las instituciones educativas tratan de difundir en quienes las frecuentan, así como los efectos que dicho contenido provoque en sus receptores" (GIMENO SACRISTÁN, I989, p I). Esta apelación a lo "cultural" ubica la problemática del currículum, tradicionalmente abordada por campo de la educación en el de la antropología, en el siguiente apartado avanzaremos sobre este aspecto.

En la actualidad, Tomáz Tadeu da Silva (1999) sostiene que el currículum es un "documento de identidad" que construye identidades a partir de diferentes enfoques sobre la sociedad, y agregamos nosotros sobre diferentes enfoques en torno al concepto de cultura. En este sentido habría diferentes visiones del currículum; una tradicional que supone una concepción conservadora y empirista de la cultura (fija, estable y heredada) donde los conocimiento se constituyen a partir de información objetiva; una tecnicista donde se enfatiza las prácticas económicas de la socieddad que construye una concepción instrumental y utilitarista de la cultura; la crítica donde el currículum refleja y reproduce las estructuras de la sociedad capitalista donde se impone o se resiste una determinada cultura, y finalmente una posestructuralista que reformula algunos análisis de la perspectiva crítica, proponiendo analizar al currículum como una práctica cultural. En línea con esta ultima perspectiva se encuentra Alicia de Alba quien caracteriza a los diferentes sujetos involucrados en la práctica curricular;

“... los sujetos de la determinación curricular (son) aquellos que están interesados en determinar los rasgos básicos o esenciales de un currículum particular. En términos generales, son sujetos

\footnotetext{
${ }^{5}$ Para Frigerio, Poggi y Tiramonti, el currículum prescripto constituye la manera de formular el contrato entre la escuela y la sociedad. Es la normativa estructurante de las instituciones educativas que delimita los aspectos de los que debe hacerse cargo la institución. No obstante, según estas autoras, toda norma deja intersticios, es decir, espacios abiertos, que permiten un margen de libertad y de creatividad (Frigerio, Poggi y Tiramonti, 1995).
} 
sociales que, si bien tienen un interés específico en relación a la orientación del currículum, en muchos casos no tienen una presencia directa en el ámbito escolar. Los sujetos sociales del proceso de estructuración formal del currículum, son aquellos que le otorgan forma y estructura al currículum, de acuerdo a los rasgos centrales perfilados en el proceso de determinación curricular. (...) Los sujetos sociales del desarrollo curricular son aquellos que convierten en práctica cotidiana un currículum. Nos referimos a maestros y alumnos (...) imprimiéndole diversos significados y sentidos y, en última instancia, impactando y transformando de acuerdo a sus propios proyectos sociales, la estructura y determinación curricular iniciales." (DE ALBA, 1994, p. I22).

En esta misma línea de análisis, en la argentina, Silvina Gvirtz y Mariano Palamidessi, sostienen que el currículum es;

\footnotetext{
“...un proceso social que se constituye a partir del cruce de diversas prácticas y dentro de un universo de significaciones particulares e históricas propias de cada institución (...) la construcción del currículum abarca el conjunto de procesos que intervienen en la definición de qué y del cómo se enseña y aprende en las escuelas. Por lo tanto, todo lo que contribuye a dar forma al qué y al cómo se enseña son considerados parte del currículum". (1998, p. 75).
}

Rescatamos esta conceptualización, que desde las Cs. de la Educación se hace, ya que nos permite plantear que el currículum, y su expresión escrita los diseños curriculares, no solo dan cuenta del "qué" se enseña, sino que hace referencia al "cómo", lo cual desde nuestro enfoque nos permite introducir las diferentes prácticas a partir de las cuales construimos conocimiento de nuestra propia sociedad.

\section{CURRÍCULUM EN CLAVE ETNOGRÁFICA: EDUCACIÓN, ESCOLARIZACIÓN Y CULTURA}

En el apartado anterior analizamos cómo las Cs. de la Educación, la Pedagogía, la Sociología de la Educación conceptualizan el currículum. Explicitamos también cómo desde diferentes enfoques se establecen relaciones conceptuales entre el currículum y diferentes perspectivas sobre la cultura y entre las teorías curriculares y una determinada conceptualización de los procesos culturales. No obstante, como sostiene Elsie Rockwell;

\footnotetext{
“...si bien muchos trabajos han abordado las relaciones entre la experiencia escolar y el entorno social, existe cierta tendencia a dejar el estudio de la interacción entre maestros y alumnos a otras disciplinas, como la didáctica y la psicología educativa y, sobre todo, el análisis del discurso. Estas disciplinas recurren de manera creciente a conceptos socio-culturales; sin embargo, suelen tratar lo cultural como atributo del contexto, de los contenidos o de los educandos, pero rara vez como una dimensión inherente a las prácticas educativas mismas." (2007, p. 176)
}

En la Argentina, al igual que en otros países con sistemas educativos estatales centralizados a nivel nacional, el proceso que denominamos educación está relacionado con otro proceso que tenemos absolutamente naturalizado y que incluso asumimos como sinónimo; la escolarización. Elsie Rockwell concibe "...la educación como un entramado de procesos de transmisión, reproducción, apropiación y transformación de objetos, saberes y prácticas culturales en contextos diversos que siempre rebasan la 
instrucción formal.” (2007, p. 175). Educación no necesariamente se correspondería con escolarización, no obstante, la autora explicita como;

"...la gran diversidad de contenidos y formas que asumen estos procesos humanos universales. Ha constatado que las dinámicas históricas de la cultura en espacios educativos, como en otros dominios - parentesco, asentamiento, trabajo, celebración, autoridad-, están sujetas a juegos y tramas de poder -legitimación, dominación, resistencia, coerción-, e involucran tanto continuidades como rupturas a lo largo del tiempo." (ROCKWELL, 2007, 176).

De esta manera, la autora no solo retoma la tradición disciplinar de la Antropología al diferenciar educación de escolarización, sino que introduce sino que introduce un enfoque sobre la cultura como parte del entramado relacional. "La visión antropológica sobre la educación en sociedades escolarizadas ha logrado esclarecer planos de conflicto, de correspondencia y de influencia mutua entre las prácticas culturales que ocurren en las escuelas y aquellas que caracterizan los entornos locales y los flujos globales." (ROCKWELL, 2007, p. 176).

A ese conjunto de prácticas, los diferentes enfoques de la antropología -en especial a partir de que Boas ${ }^{6}$ rechazó el evolucionismo social de Tylor- las han conceptualizado como "cultura", "lo cultural", "procesos culturales", "fenómenos culturales", "objetos culturales", etc. ${ }^{7}$. Actualmente hay ciertos acuerdos al interior de la antropología respecto a estos conceptos, Esteban Krotz los sintetiza con claridad;

“.. . la cultura no es sólo lo que se encuentra en museos, teatros y bibliotecas, sino también lo que está en las calles y las casas, la opción por una influencia cultural con alternativas culturales tiene que ver con la identidad colectiva de una población, o sea, de cómo ésta ve la vida y quiere vivirla y qué sentido encuentra en ella. Pero esto remite enseguida a la estructura del poder vigente en el seno de esta población: ¿Quién tiene la capacidad de reconocer alternativas, quién puede decidir sobre cuál se acepta o no? y, en dado caso, icómo?" (KROTZ, 1994, p. 7).

Analicemos cómo en algunos documentos oficiales sobre el currículum se hacen referencia a la relación entre educación, escolarización y cultura. El portal EDUCAR, del Ministerio de Educación de la República Argentina, destinado a directivos, docentes, profesoras/es sostiene que:

\footnotetext{
"El currículum integra un conjunto de objetivos, contenidos y criterios pedagógicos y didácticos. Diseñado desde el Estado, reglamenta los saberes que las escuelas, colegios, centros de formación y otros establecimientos educativos deben transmitir a las nuevas generaciones. Estos lineamientos surgen del consenso entre el Ministerio de Educación de la Nación y el Consejo Federal de Educación, a fin de garantizar una calidad educativa, asegurando la integración nacional con sentido federal. De este modo, el currículum supone una construcción colectiva que delinea, entre otras cosas, los núcleos de aprendizajes prioritarios (NAP). El currículum es un documento público que tiene un carácter normativo y establece contenidos comunes. Se instaura, así como un guion estratégico que desde el Estado garantiza condiciones de igualdad e integración para el sistema educativo nacional." (Portal EDUCAR).
}

\footnotetext{
${ }^{6}$ Franz Boas, fue un antropólogo norteamericano de origen alemán quien sostuvo que la cultura es el producto de particularidades históricas y sociales.

7 Si bien consideramos que todas estas categorías son válidas en esta presentación utilizaremos "cultura".
} 
En el extracto se hace explícito el lugar "nacional", "normativo" y "estatal" del sistema educativo. En el Marco General de Política Curricular del Ministerio de Educación de la Pcia. de Bs.As. se plantea que:

"La política curricular de la provincia de Buenos Aires se sostiene a partir de una concepción de curriculum entendido como "síntesis de elementos culturales (conocimientos, valores, costumbres, creencias, hábitos) que conforman una propuesta político-educativa” (De Alba, Alicia, 1995, p. 59). (...) En tanto propuesta histórica, cultural, social y políticamente contextuada el currículum constituye una selección arbitraria y transitoria del patrimonio cultural. La noción de arbitrariedad hace referencia al carácter situado de la selección de algunos contenidos culturales considerados valiosos, necesarios y significativos para ser enseñados a todas las personas. Dicha selección se realiza para y desde una realidad socio-histórica y cultural compleja y diversa, en el marco de una política pública que procura la construcción de futuros deseados y con un determinado desarrollo científico y tecnológico." (Marco General de Política Curricular - ABC, 2007, p | 5).

Es interesante destacar cómo desde la política educativa de la Provincia de Buenos Aires se sostiene que lo que vincula "currículum" con "cultura" es la idea de "síntesis de elementos culturales", y explícitamente se aclara lo que se entiende por "culturales": "conocimientos, valores, costumbres, creencias, hábitos". Esta concepción de la cultura nos recuerda las conceptualizaciones esencialistas de la antropología clásica donde determinados rasgos, específicos y delimitados, son considerados como la expresión de las características de un determinado grupo humano. Esta concepción de "cultura" asimismo supone una forma de construir conocimiento sobre la propia sociedad a partir de construir límites y rasgos objetivos que adquieren valor explicativo en sí mismo, ignorando las relaciones de hegemonía y subalternidad que los constituyen. Como sostiene Susan Wright estas definiciones "implican una toma de posición y son políticas, y por ende un recurso que antropólogos y otros pueden usar para el establecimiento de procesos de dominación y marginación o el desafío a ellos.” (1988, p. 16). A tal punto queda clara la "politización de la cultura" que en el mismo extracto podemos leer como la "arbitrariedad" es asumida para delimitar los "contenidos culturales" de lo que se considera necesario enseñar en un determinado momento histórico. En esta misma línea se inscriben los Diseños Curriculares de la Provincia de Buenos Aires para la Educación Secundaria, pero haciendo referencia a "la cultura":

"La selección de los conocimientos a ser enseñados en este nivel es un recorte de la vastedad de
conocimientos, experiencias y saberes que forman parte de la cultura. Atendiendo a la necesidad
de contar con un repertorio posible de ser enseñado en la escuela, la propuesta curricular que se
presenta se dirige no sólo a que los alumnos/as adquieran esos saberes, sino que además puedan
reconocerlos como aquellos conocimientos necesarios, pero a la vez precarios, inestables y
siempre cambiantes, producto del constante movimiento de la ciencia, las artes y la filosofía, al
que tienen el derecho fundamental de acceder como sujetos sociales." (Diseño Curricular para I
año $\left(7^{\circ} \mathrm{ESB}\right)$ Marco General, DGE, 2006, p. I I)

En los Diseños Curriculares de la Ciudad de Buenos Aires se explicita claramente que el objetivo de la escolarización primaria es "... la apropiación de la cultura (...) garantizar el acceso a saberes, prácticas y experiencias culturales relevantes para la realización integral de las personas". (Diseño Curricular para la Escuela Primaria. Primer Ciclo, CABA, 20 I2, p 36). En el documento Marco General de la Nueva Escuela 
Secundaria que el Ministerio de Educación de la CABA establece para los Diseños Curriculares no se hace referencia al concepto de cultura, pero sí se establecen un conjunto de prácticas que establecen los objetivos de dichos diseños.

"El Diseño Curricular del Ciclo Orientado de la Escuela Secundaria Orientada es el resultado del trabajo conjunto y coordinado del Ministerio de Educación de la Ciudad Autónoma de Buenos Aires en consulta con los diversos actores del sistema educativo de la jurisdicción y apunta a la mejora continua de los niveles de inclusión educativa (permanencia, avance y egreso de los estudiantes) garantizando niveles de calidad que permitan su desarrollo personal y el ejercicio de una ciudadanía responsable. Se propone ser un marco en el cual se inscriban prácticas de enseñanza y aprendizaje que construyan nuevos conocimientos y que potencien el desarrollo máximo de las habilidades y competencias en un marco de respeto por la diversidad y de preparación para la vida futura. Las definiciones presentadas en este marco pedagógico requieren la necesaria contextualización para ser puestas en juego en cada aula y de acuerdo con cada realidad institucional. En este sentido, es importante el compromiso de la comunidad educativa en el logro de los aprendizajes de los estudiantes, considerados como centro y destinatario de las acciones de mejora. De ahora en adelante, queda a las escuelas la tarea de elaborar su Proyecto Curricular Institucional, sobre las bases aquí plasmadas. (Marco pedagógico Ciclo Orientado del Bachillerato, CABA, 20I5, p. 65).

"Trabajo conjunto", "consulta”, "mejora continua”, "inclusión”, "potencia”, "compromiso" etc. son algunas de las prácticas que promueven los diseños curriculares de la CABA para la Nueva Escuela Secundaria. Es interesante analizar dichas prácticas como expresión de una determinada política, como expresión de una clase social ${ }^{8}$ que construye una particular posición de clase en un discurso legítimo en un determinado momento histórico y social. Como sostiene Susan Wright en

“... su forma más segura, una ideología aparece como hegemónica. Esto es, se torna tan naturalizada, dada por hecho y 'verdadera' que las alternativas están fuera de los límites de lo imaginable (...) en su dimensión hegemónica, la cultura aparece como coherente, sistemática y consensuada. Procura aparecer como un objeto, una cosa más allá de la acción humana, no ideológica en lo más mínimo: en pocas palabras, como la vieja idea de cultura auténtica." (WRIGHT, 1998, p. 6).

Esta perspectiva cosificada del concepto de cultura, que vemos en los diferentes documentos oficiales "...no solo está íntimamente vinculada a las relaciones económicas básicas, sino que es algo que hay que crear activamente, en especial si un grupo o una clase aspira a la hegemonía" (CREHAM, 2004, p. 59) Los documentos curriculares -y los contenidos- hacen uso de una perspectiva esencialista de la cultura que posibilita presentar una determinada perspectiva de clase sobre la sociedad como hegemónica 9 .

\footnotetext{
${ }^{8}$ Este razonamiento respecto a la dinámica de clase, también puede ser aplicado a las relaciones de género o étnicas de cara a entender cómo y por qué se organizan el currículo privilegiando determinadas perspectivas respecto a la enseñanza.

${ }^{9}$ Este mismo tipo de razonamiento podemos establecer para las relaciones que en un determinado momento histórico y en una determinada sociedad construimos con el conocimiento. Las relaciones entre educación-conocimiento-escolarización no se presentan en todas las sociedades de la misma manera, e incluso en una misma sociedad a lo largo de la historia esta relación se va transformando. El conocimiento no es inmutable, sino que, en tanto recurso epistémico socialmente disponible, es construido a partir de un proceso que involucra prácticas sociales.
} 


\section{UNA PROPUESTA DE ABORDAJE: LA CATEGORÍA LOCAL}

Sostuvimos en el apartado anterior que el currículum es parte de la política educativa que un determinado Estado lleva delante en un contexto histórico y social. Es en los documentos escritos del currículum -diseños curriculares- donde se expresan los conocimientos legitimados en un determinado momento histórico para garantizar la escolarización de los sujetos. Determinados posicionamientos de clase social sobre el mundo del trabajo, el ejercicio de la ciudadanía, las formas de gobierno, etc. son transmitidos de manera hegemónica en los diseños curriculares. Igualmente, analizamos cómo dichas perspectivas de clase son invisibilizadas a partir de un uso político -o ideológico-del concepto de "cultura". Uso que se presenta oculto bajo el carácter explicativo que se le otorga al concepto de "cultura". De esta manera las teorías o argumentaciones formuladas "bajó" dicho concepto se van entretejiendo en el sentido común y pasan a formar parte de las explicaciones y las teorías presentes en el sentido común (ARRIBAS, BOIVIN Y ROSATO, 2004). Estos efectos de teoría relacionan representaciones sociales y práctica cotidiana marcando anticipadamente un "orden", una "jerarquía" entre "nosotros" y los "objetos sociales". Finalmente, el currículum -como vimos en el primer apartado- es parte de un campo disciplinar, el de las Ciencias de la Educación y la Pedagogía; por ende, supone teorías curriculares. Dentro de estos metadiscursos teóricos, se estudian las conceptualizaciones y concepciones curriculares, los paradigmas de investigación y práctica curricular, y todo lo relativo a supuestos científicos y técnicos involucrados en las concepciones curriculares. Estas conceptualizaciones técnicas del currículum hegemónicas proponen un aprendizaje descontextualizado al aislar y deslegitimar las prácticas situadas que despliegan otros sujetos escolares; como pueden ser docentes, estudiantes, etc. (Lave, 200I). Si bien algunas de las teorías curriculares, enmarcadas en lo que se conoce como "pedagogía critica", explicitan el carácter ideológico del currículum siguen comprometidas

\footnotetext{
"....con la tarea de "develar" y de "transformar la práctica y la conciencia de los sujetos estudiados. A veces hay algo de misionero, algo de etnocéntrico en esta postura, que no creo compatible con la tarea de hacer etnografía. Cuando se parte de una idea preconcebida de lo que es una práctica "buena", y se denuncian las prácticas "malas", no es posible acercarse al conocimiento local que hace inteligibles aquellas prácticas que no son como quisiéramos que fueran." (ROCKWELL, 1994 p69)
}

El currículum es una de las prácticas posibles a partir de las cuales una determinada sociedad se conceptualiza a sí misma. Esta conceptualización hace uso de una determinada perspectiva sobre "la cultura" para invisibilizar su carácter ideológico de clase permitiendo organizar la transmisión, apropiación y producción de los conocimientos legitimados en un determinado momento histórico, a las subsiguientes generaciones. Analizar desde una perspectiva antropológica al currículum implica desnaturalizar los usos que en la cotidianeidad se hace de dicho "objeto social". Supone asumir el "extrañamiento" como punto 
de partida necesario de toda práctica antropológica, sea esta de investigación extensión o docencia. Esta práctica de extrañamiento permite analizar al currículum como un "objeto social" que enuncia una determinada política estatal, una perspectiva de clase, un conjunto hegemónico de conocimientos disciplinares y un entretejido de prácticas desplegadas por los sujetos al construir conocimiento en la cotidianeidad escolar. En línea con estos razonamientos es que proponemos analizar al currículum como un categoría local.

Una categoría, en tanto establece criterios de clasificación para establecer partes o unidades, y local dado que adjetiva dichas clasificaciones en un marco temporal y espacial determinado. Al decir de Rockwell (1994) en la etnografía "... el trabajo teórico no nos aleja necesariamente del conocimiento local, permite, más bien, el acercamiento a los significados culturales locales que los actores sociales otorgan a los procesos en los que participan." (p. 62). Debido a esto es que, al inicio de este artículo, hicimos referencia a las construcciones teóricas que a lo largo de la historia disciplinar construyeron diferentes referentes de la antropología. Dichas construcciones conceptualizaban las prácticas que en determinadas sociedades se despliegan para construir conocimiento sobre si mismas; es en este conjunto de prácticas que ubicamos al currículum, y es por lo cual proponemos abordarlo como una categoría local.

En primer lugar, puesto que es una categoría propia de las teorías curriculares y el campo disciplinar de la Didáctica y/o la Pedagogía, y no de la Antropología. En este sentido los diseños curriculares expresan las prácticas a partir de las cuales, las nombradas disciplinas, conceptualizan el mundo social. El currículum con los que directivos, docentes y profesores trabajan cotidianamente en las escuelas ${ }^{10}$, contienen los conocimientos que se consideran legítimos en un determinado momento histórico y social pero "organizados", "secunciados" y "jerarquizados" por un campo disciplinar. Al abordar el currículum como una categoría local nos distanciamos de las explicaciones que los propios sujetos construyen sobre dicho objeto, lo cual nos permite analizar, desde qué prácticas de construcción de conocimiento y posicionamientos políticos se conceptualiza la propia sociedad, como analizamos en el apartado anterior respecto a las concepciones de "cultura" a la que se apela en los documentos oficiales. Asimismo al hacer uso del currículum, ya sea desde quienes participan en los diseños hasta quienes se vinculan en la cotidianeidad escolar, se expresa un conocimiento local que construye "realidades" sociales, en lugar de reflejarlas simplemente como se pretende sostenerse desde la teoría curricular. Abordar el currículum como categoría local nos permite visibilizar las huellas históricas presentes en las diferentes concepciones teóricas que organizan el conocimiento que se transmite, apropia y produce. Lo cual nos posibilita examinar los diseños curriculares como parte de una política educativa que

\footnotetext{
${ }^{10}$ Lo mismo podríamos sostener con relación a los diseños curriculares que fueron definiendo lo que hoy es la antropología en los diferentes espacios académicos.
} 
“... no pueden analizarse como objetos estáticos, dado que su diseño, implementación y aplicación involucra múltiples sentidos y prácticas (...) nos interesa documentar los procesos sociales que expresan el carácter social e histórico de los sentidos atribuidos al estado y de las prácticas desplegadas en las instituciones estatales: parafraseando a Rockwell, lejos de constituir simples instrumentos usados por el estado para moldear a los sujetos, las escuelas se convierten en sitios donde diversas representaciones de las políticas educativas entran en juego, son apropiadas, resistidas y negociadas" (HIRSCH y RÚA, 2009, p 2 I3).

En segundo lugar, el currículum expresa un determinado orden disciplinar, un conjunto de teorías curriculares que "...transforman las «sensaciones» incoherentes y fragmentarias de quienes viven una determinada posición de clase en un discurso coherente y razonado del mundo tal como se percibe desde esa posición." (CREHAM, 2004, p. 59). Este "discurso coherente y razonado" en el contexto educativo, y en nuestra sociedad occidental está expresado en los diseños curriculares ${ }^{11}$, y es conceptualizado por las teorías curriculares. Desde los enfoques que intentan definir técnicamente hasta los actuales que problematizan sus usos, todas las teorías curriculares asumen al currículum como un objeto integrado a su campo disciplinar. Es importante abordar a las teorías curriculares como "manifestaciones históricas de la formación sociocultural de la que somos participantes y con las que tenemos conexiones concretas de diverso tipo." (Lave, 200I, p.4I).

Finalmente, abordar al currículum como una categoría local nos permite problematizar las prácticas que se construyen en torno al currículum, y en este sentido es que consideramos necesario analizar los usos que se hace del currículum en la cotidianeidad escolar. Éste es el aspecto central para nosotros, puesto que el currículum supone complejos procesos de interacción entre las teorías curriculares, las políticas curriculares, los diseños curriculares y los sujetos que transmiten, se apropian y producen conocimiento respecto a la propia sociedad. Es en ese campo de mediaciones donde se construyen las prácticas concretas que se materializan localmente en la cotidianeidad escolar.

Analizar el currículum como una categoría local posibilita analizar el entramado relacional en el que dicho objeto social se despliega. Nos permite ubicar las teorías y los diseños curriculares como parte del proceso social a partir del cual una sociedad en un determinado momento histórico construye conocimiento respecto a sí misma. Analizar los usos históricos y sociales que se hacen de las teorías y los diseños curriculares posibilita desnaturalizar la categoría de currículum. Al conceptualizar el currículum como una categoría local creamos un distanciamiento que posibilita problematizar el entramado relacional que le otorga sentido. Esto nos permite analizar el currículum como producto de la capacidad creadora que todo colectivo social despliega al generar objetos explicativos de su propia sociedad. Las categorías locales condensan la lógica sociocultural que los sujetos despliegan respecto a una determinada

\footnotetext{
II Gabriela Novaro (2005) en "Nacionalismo escolar y migraciones en educación: de las "hordas cosmopolitas" a los "trabajadores competentes" analiza como en los diseños curriculares para la educación primaria -pero también en las editoriales y los usos en las aulas- se privilegian ciertas "narraciones" escolares en torno a la formación y organización de la nación y el proceso migratorio por sobre otras.
} 
problemática social, el currículum objetiva la lógica sociocultural de la clase social que detenta la hegemonía, mediante un proceso de descontextualización de las prácticas que producen las teorías y los diseños curriculares, proceso que simultáneamente legitima la "generalización" de un "punto de vista" por sobre sobre otros respecto a la propia sociedad.

Enseñar antropología implica dar cuenta de sus principales posicionamientos teóricos, epistemológicos y metodológicos, supone dar cuenta del contexto histórico y social en el cual se desarrolló la disciplina. Enseñar a enseñar antropología, implica analizar la relación que hemos construido con el campo científico en general: en nuestro caso la relación con la Didáctica.

\section{REFERÊNCIAS}

ACHILL, E. (2008). Investigación y Formación Docente. Laborde.

APPLE, M. W. (2008). Ideología y currículo. Akal.

Arribas, V; Boivin, M y Rosato, A. (2004). Constructores de Otredad. Una introducción a la Antropología Social y Cultural. Antropofagia.

CAMILLONI, A. (1997). El campo de la didáctica y su relación con los contenidos, en Novedades Educativas, $n^{\circ} 51$. Reseña

CERLETTI, L. Y RÚA, M. (20 I7). Antropología de la Enseñanza: conocimientos y experiencias. En Cerletti, L. y Rúa, M. (Comps.), La Enseñanza de la Antropología. Colección Libros de Cátedra. Editorial de la Facultad de Filosofía y Letras, Buenos Aires.

CREHAN, K. (2004). Gramsci, cultura y antropología. Bellaterra, Barcelona.

DA SILVA, T. (1995). Escuela, conocimiento y currículum, Buenos Aires, Miño y Davila.

DE ALBA, A. ( 1994). Curriculum: Crisis, mito y perspectiva, Miño y Davila.

DÍAZ BARRIGA, Á. (2003). Currículum. Tensiones conceptuales y prácticas. Revista Electrónica de Investigación Educativa, 5 (2). Consultado el día de mes de año en:

http://redie.ens.uabc.mx/vol5no2/contenido-diazbarriga.html

Dirección General de Cultura y Educación. Diseño Curricular para la Educación Secundaria: $1^{\circ}$ año ESB / coordinado por Ariel Zysman y Marina Paulozzo - 2a ed. - La Plata: Dir. General de Cultura y Educación de la Provincia de Buenos Aires, 2006.

Dirección General de Cultura y Educación. Marco General de Política Curricula. - La Plata: Dir. General de Cultura y Educación de la Provincia de Buenos Aires, 2007.

Diseño curricular nueva escuela secundaria de la Ciudad de Buenos Aires, marco general; dirigido por Gabriela Azar. - Ia ed. - Ciudad Autónoma de Buenos Aires: Ministerio de Educación del Gobierno de la Ciudad Autónoma de Buenos Aires. Dirección General de Planeamiento e Innovación Educativa. 20 I 5. 
Diseño curricular para la escuela primaria: primer ciclo de la escuela primaria, educación general básica / dirigido por Silvia Mendoza. - Ia ed. I a reimp. - Ciudad Autónoma de Buenos Aires: Ministerio de Educación del Gobierno de la Ciudad Autónoma de Buenos Aires, 2012.

EISNER, E. (1985). Procesos cognitivos y currículo. Barcelona, Martínez Roca.

EZPELETA, J. Y ROCKWELL, E. "Escuela y clases subalternas". En: Educación y clases populares en América Latina. ROCKWELL e IBARROLA (comps). IPN, DIE. México. 1985.

FELDMAN, D. (2010). Enseñanza y escuela. Buenos Aires, Paidós.

FOUCAULT, M. El orden del discurso. Tusquets, Bs. As. 2005.

FRIGERIO, G.; POGGI, M. Y TIRAMONTI, G. (1995). Las instituciones educativas. Cara y Ceca, Buenos Aires, Troquel.

GIMENO SACRISTÁN, J. (1989). El currículum: una reflexión sobre la práctica, Madrid, Morata.

GIMENO SACRISTÁN, J. (20I0). "¿Qué significa el currículum?” En Saberes e incertidumbres sobre el curriculum. Madrid.

GVIRTZ, S. Y PALAMIDESI, M. (1998). El ABC de la tarea docente: currículum y enseñanza. Buenos Aires: Aique.

HERNÁNDEZ, F. Y VENTURA, M. ( 1992). La organización del currículum por proyectos de trabajo. Barcelona: I.C.E de la Univeridad de Barcelona.

HIRSCH, M y RÚA, M; (2009). "Reflexiones en torno al "derecho a la educación": entre las políticas educativas y la cotidianeidad escolar". En Actas del Segundas Jornadas de Antropología Social del Centro Bonaerense. "Antropología y crisis global: Desafíos para una interpretación desde el Sur". UNICENFacultad de Ciencias Sociales. Olavarría.

JACKSON, P.W. (1968) La vida en las aulas, Morata.

KROTZ, E. (1994). "Cinco ideas falsas sobre cultura”. En Revista de la Universidad Autónoma de Yucatán, volumen 9, de octubre diciembre de 1994, núm. 191, págs. 31-36.

LAVE, J. (200 I). "La práctica del aprendizaje”. En Estudiar las prácticas. Perspectivas sobre actividad y contexto. Amorrortru, Buenos Aires.

NOVARO, G. (2005). "Nacionalismo escolar y migraciones en educación: de las "hordas cosmopolitas" a los "trabajadores competentes"”. En Domenech, E. (comp.), Migraciones contemporáneas y diversidad cultural en la Argentina. Universidad Nacional de Córdoba - Centro de Estudios Avanzados.

ROCKWELL, E. ( 1 994). "La etnografía como conocimiento local”. En Rueda, M; Delgado Ballesteros, G. y Jacobo, Z. (Coordinadores). La etnografía en educación: Panorama, Prácticas y Problemas. Centro de Investigaciones y Servicios Educativos-UNAM. Circuito Exterior, Ciudad Universitaria, Delegación Coyoacán, México.

ROCKWELL, E. (2007). "Huellas del pasado en las culturas escolares". Revista de Antropología Social. Universidad Complutense de Madrid, VI6, pp 175-212. 
RÚA, M. (2017) "Un análisis antropológico de los procesos de construcción de conocimiento: Las prácticas de escritura en la trama escolar". En Revista Educación, Lenguaje y Sociedad. Universidad Nacional de La Pampa. Vol XIV. No I4. (ISSN 1668-4753).

SINISI, L., CERLETTI, L. Y RÚA, M. (20I7) "La enseñanza de la Antropología y la formación de profesores”. En Cerletti, L. y Rúa, M. (Comps.), La Enseñanza de la Antropología. Colección Libros de Cátedra. Editorial de la Facultad de Filosofía y Letras, Buenos Aires. 2017

TYLER, R. (1973). Principios básicos del currículo. Buenos Aires: Troquel.

WRIGHT, S. ( 1998) "La politización de la "cultura"”. En Anthropology Today Vol. I 4 No I, Febrero de 1998. Traducción de Florencia Enghel. Revisión Técnica de Mauricio F. Boivin y Julieta Gaztañaga. 